\section{PLATFORM PRESENTATIONS}

\section{1}

THE TRIGEMINOCERVICAL REFLEX IN MIGRAINE

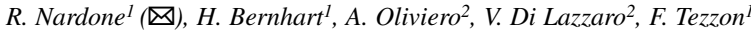

${ }^{I}$ Department of Neurology, F. Tappeiner Hospital, Merano (BZ), Italy; ${ }^{2}$ Institute of Neurology, Catholic University, Rome, Italy

Recent theories about migraine pathogenesis have emphatized the role of the trigeminal system in the pathogenesis of migraine attacks. The local vasodilatation of intracranial extracerebral blood vessels and the consequent stimulation of surrounding trigeminal sensory nerve pain pathways are proposed to be the key mechanisms underlying the generation of headache pain associated with migraine. The "triptan" anti-migraine agents are serotoninergic agonists that act by inhibiting nociceptive transmission at 5-HT1D receptors on peripheral trigeminal sensory nerve terminals in the meninges and central terminals in brainstem sensory nuclei. Because there are no structural disturbances in migraine, only physiologic studies can provide insight into the underlying mechanisms. The trigeminocervical reflex could be a suitable parameter for evaluating the trigeminal system in migraine. Recently, short latency responses were recorded in tonically active neck muscles after stimulation of branches of the trigeminal nerve. These trigeminocervical reflexes consist of a positive/negative wave in the average unrectified electromyogram, corresponding to a short period of inhibition of voluntary motor unit firing. To determine whether blink reflex and trigeminocervical reflex are useful parameters in the study of migraine, we evaluated 10 patients with migraine with aura and in 10 patients with migraine without aura, before and after the administration of triptans; these patients were compared with 10 healthy subjects.

The trigeminocervical response was abnormal in 7 patients with migraine with aura and in 6 patients with migraine without aura. The latency of the R 2 component of the blink reflex was significantly different only in 3 patients with migraine with aura and in 3 patients with migraine without aura. Moreover, the blink reflex was also altered in 2 control subjects. After administration of a triptan, the trigeminocervical response was altered in 4 patients with migraine with aura and in 4 patients with migraine without aura: these patients showed a poor response to therapy.

The trigeminocervical reflex is the most sensitive parameter for evaluating the involvement of the trigeminal system in migraine attacks. Their assessment may provide prognostic information. These findings support the concept that trigeminal afferents and polysynaptic pathways in brainstem may be altered in migraine. Our study confirms that a dysfunction of the control system on the trigeminal networks may predispose to migraine attacks.

\section{2}

EXPERIMENTAL MODEL DESCRIBING HOW TO APPLY THERAPEUTIC AND DIAGNOSTIC GUIDELINES TO MEDICAL CASES OF MIGRAINE IN THE REGION OF CALABRIA: EPIDEMIOLOGY, QUALITY OF LIFE AND ECONOMIC ASPECTS

R. Iannacchero ${ }^{I}(凶)$, U. Cannistrà ${ }^{2}$, F. Fonte ${ }^{3}$, E. De Caro $^{2}$

${ }^{I}$ Territorial Neuromotor Rehabilitation Unit, ASL6, Lamezia Terme (CZ), Italy; ${ }^{2}$ Department of Neurology, Pugliese Ciaccio Hospital, Catanzaro, Italy;

${ }^{3}$ Department of Neurology, ASLsp5, Crotone, Italy

Introduction When you hear of migraine, you shouldn't think of it simply as a headache. In fact, it is a frequent trouble affecting the quality of life of more and more people. This system allows getting an univocal, comparable and testable diagnosis among which migraine represents the most frequent case of primary headache. Consequently the disability becomes of chronic kind affecting the quality life of patients.

Epidemiology and analysis of costs In Calabria, the economic and social issues of the illness are underestimated at the moment because of its episodic nature and of limited requests for specialist medical treatments, which adds to a lack of headache research centres. From an economic and social point of view, the evaluation of an illness involves an assessment of its direct and indirect costs and of the quality life which in its turn allows one to evaluate the influence of disease on man. The "direct costs" are connected to the economic resources necessary for medical treatment in the three-phase process of prevention, diagnosis, and cure. The "indirect costs" are linked to the fact that a person works less than usual because he feels bad, and last but not least there are the intangible costs which are of psychological and social nature in that they affect the family life of patients and their social relationships. The quality life of a person suffering from chronic headache is strongly conditioned. Therefore even if the patient doesn't feel that his expectation of living has shortened, his well being proves to be strongly limited. Operative proposal In Calabria, one of the most relevant problems in the health department is to control the demand of medical services. As far as medical services are concerned, the scarcity of resources and the funding system are calling for a direction of the supply-demand relationship. It follows that it is difficult from the part of local health units to work out a plan and to make right choices. Moreover there are other issues to face up to, such as the problems of equity, approriateness and management of demand and of strengthening health. Therefore, the role played by the general practitioner is fundamental in drawing and addressing diagnostic and therapeutical flow-charts. This issue links to that concerning the problem of bringing medical services nearer to people by developing and by conferring value and quality to the protection of health in all its forms of organization. In light of what has been proposed and experienced in a pilot solution, some surgeries should be set up for the diagnosis and cure from a neurologic, anesthesiologic and psychiatric viewpoint.The activities of surgeries will work together with those of the directors working within the same local health units in order to fulfill an integration between hospital and county and with the aim of carring out an economic collaboration between the parts involved.

Discussion The aim of the regional coordination known as S.I.S.C. Calabro is to point out, starting from a regional model, how to apply the most suitable management strategies to local health units. The strategies will have to be based on a regional management model so as to provide the social and economic issue with a sound basis. The regional models show in real terms the way creating and providing other medical centres and treatment seivices besides those usually given by hospitals.

\section{3}

\section{ASSOCIATION BETWEEN MIGRAINE AND PERSONALITY CHANGES. A LONGITUDINAL STUDY}

F. Mongini (更), R. Keller, A. Deregibus, F. Raviola, T. Mongini, M. Sancarlo Department of Clinical Pathophysiology, Headache and Facial Pain Unit, University of Turin, Turin, Italy

The purpose of this work was to study (1) if an association exists between migraine and personality changes and (2) if this association is unidirectional (that is, migraine causing personality changes) or bidirectional. The study consecutively enrolled 60 patients ( 4 men and 56 women) with migraine with or without aura (time 0 ). They were given a diary in which they were asked to record for one month, on a daily basis, the occurrence, severity (score 1 to 5), and duration of the headache episodes. The MMPI and the STAI, X1,2 were administered. The patients were then treated with antimigraine drugs, NSAIDs and antidepressants, as needed. They were reexamined six years later (time 1) and the following tests were administered: MMPI-2, STAI Y1,2, and Beck depression inventory. A structured interview was also performed using the SCID 1. If still present, the headache was monitored for three months. The patients were then distributed in two groups: Group 1, with pain improvement (at least $50 \%$ reduction of the headache frequency) and Group 2, with no pain improvement. All data collected at time 0 and time 1 were assessed separately for the two groups and statistically analysed (Student's $t$ and Fisher's exact tests).

The following items were found to be lower in Group 1: STAI 1,2 and all MMPI scores at time 0 (significantly for depression, psychopathic deviation, paranoia, psychasthenia, schizophrenia and social introversion); all STAI 1,2, MMPI and Beck scores at time 1 (all significantly, except hypomania); prevalence of major depression or dysthymic disorder. No difference in the pain parameters at time 0 was found between the two groups.

We conclude that: (1) an association exists between personality changes and migraine, and (2) that this association seems to be bi-directional.

\section{4}

\section{COMORBIDITY OF MIGRAINE WITH AURA WITH MOOD DISORDERS: AN EPIDEMIOLOGICAL STUDY IN GENERAL POPULATION}

D. Cologno (凶), P. Torelli, F. Brindani, G.C. Manzoni

Headache Centre, Institute of Neurology, University of Parma, italy

Psychiatric disturbances are often associated with headache, most notably with daily chronic headache and tension-type headache. However, associations with migraine without aura (MO) and with migraine with aura (MA) have also been suggested by several authors. As part of a prospective epidemiological study of MA in the general population of the town of San Severo in Southern Italy beginning in June 2000 with a one-year follow-up, our group investigated the comorbidity of MA with mood and anxiety disorders. Diagnosis of the psychiatric disturbances was based on a structured interview using the MINI 4.4. version of the SCID questionnaire prepared according to DSM-IV conventions. Our initial sample consisted of 2000 persons aged 18-65 years, selected at ran- 
dom from the general population. All persons were mailed a questionnaire with the necessary items for MA diagnosis. The total number of people who accepted to participate in the study and handed in their questionnaires duly filled in was 1388 , including 844 women and 544 men. Among this sample, we found 20 MA sufferers ( 12 women and 8 men). By comparing data from the MINI 4.4. questionnaire between the $20 \mathrm{MA}$ patients and a non-MA control group of 40 subjects ( 24 women and 16 men), selected from the same population and sex- and age-matched ( \pm 2 years) to the patient group, we found: (1) major depressive episodes (over the last 2 weeks) in $25.0 \%$ of both MA patients and non-MA controls; (2) lifetime panic attacks in $25.0 \%$ of MA patients and $27.5 \%$ of controls; (3) current episodes of agoraphobia in $35.0 \%$ of both MA patients and controls; (4) agoraphobia without panic attacks in $20.0 \%$ of both MA patients and controls; and finally, (5) generalized anxiety disorder (over the last 6 months) in $40.0 \%$ of MA patients and $50.0 \%$ of controls. As the MA sufferers identified so far were so few in number, we are not able to proceed with statistical analysis. However, judging from percentage figures, there does not seem to be any significant difference between MA patients and controls. Our preliminary results appear to be at a variance with the conclusions reached by other authors.

\section{5}

QUALITY OF LIFE IN PATIENTS WITH MIGRAINE WITHOUT AURA AND WITH TRANSFORMED MIGRAINE

D. D'Amico, S. Usai, A. Solari, L. Grazzi, A. Rigamonti, M. Leone, G. Bussone (匈) National Neurological Institute C. Besta, Via Celoria 11, Milan, Italy

Several studies reported that migraine is characterized by a reduction in quality of life. Few data exist about the functional impact of chronic daily headaches. Among these forms, transformed migraine (TM) refers to a daily or almost daily headache developing from a previous episodic migraine. A group of 68 patients with migraine without aura (diagnosis according to the IHS criteria) and a group of 65 patients with TM (diagnosis according to Silberstein, et al, 1996) completed the SF-36 questionnaire. SF-36 scores at each scale in the studied patients were compared with normative data for the Italian population (Student's $t$ test and the post hoc Bonferroni method); the same methods were used to assess if any significant difference between the 2 diagnostic groups was present. Compared to normative data, patients with migraine had significantly lower scores in RP, BP, SF $(p<0.0001)$ and RE $(p=0.02)$. TM patients had significantly lower scores $(p<0.0001)$ on all SF-36 scales but one (VT). Furthermore, in patients with TM the scores of most scales (PF, RP, SF, $\mathrm{RE}, \mathrm{MH}$ ) were significantly lower than those found in migraine patients. Our results clearly indicate that migraine has a negative influence on patients' functioning. In those patients with chronic daily headache evolving from a previous migraine (TM), quality of life was even more severely affected in different roles.

\section{6}

\section{EFFECT OF RIZATRIPTAN AND OTHER TRIPTANS ON FUNCTIONAL DISABILITY IN MIGRAINE}

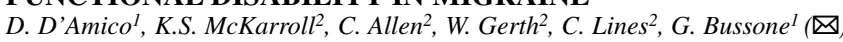
${ }^{1}$ C. Besta National Neurological Institute, Milan, Italy; ${ }^{2}$ Merck Research Laboratories, West Point, NY, USA

Migraine is associated with impairment in daily activities. The reduction of functional disability has been identified as one of the main goals of the acute anti-migraine therapy by the recently published guidelines proposed by the US Headache Consortium. In this study the efficacy of rizatriptan versus other triptans on headache-related functional disability was evaluated. Data from 5 headto-head randomized, double-blind, placebo-controlled comparative trials were used. All the enrolled patients had an IHS diagnosis of migraine, with 1-8 attacks/month, and were instructed to take the study medication when pain was moderate-severe, using an escape medication at least 2 hours after dosing. A 4 grade scale was used to assess functional disability (normal ability to function, daily activities mildly impaired, daily activities severely impaired, unable to do activities and requiring bedrest). Data were analyzed using logistic regression models. The main endpoint was return to normal function at 2 hours from dosing. Most patients had high disability during their attacks (patients who were severely disabled and patients requiring bedrest, $76 \%-97 \%$ of the total sample). At 2 hours after dosing, the percentage of patients who returned to normal functioning in the 5 comparative trials were as follows: $10 \mathrm{mg}$ rizatriptan vs. $10 \mathrm{mg}$ sumatriptan, $39 \%$ vs. $32 \%(p=0.02) ; 10 \mathrm{mg}$ rizatriptan vs. $50 \mathrm{mg}$ sumatriptan, $47 \%$ vs. $43 \%$ ( $p=0.03$ ); $10 \mathrm{mg}$ rizatriptan vs. $25 \mathrm{mg}$ sumatriptan, $48 \%$ vs. $36 \%(p=0.001) ; 10 \mathrm{mg}$ rizatriptan vs. $25 \mathrm{mg}$ naratriptan; $39 \%$ vs. $22 \%$ $(p=0.001)$; and $10 \mathrm{mg}$ rizatriptan vs. $25 \mathrm{mg}$ zolmitriptan, $45 \%$ vs. $36 \%$ $(p=0.008)$. In conclusion, rizatriptan was significantly more effective than all other triptans in reducing disability, allowing more migraine patients to return to normal function than other drugs of the same class. There was no significant treatment-by-baseline disability interactions, indicating that differences were consistent across the differing disability levels at baseline.

\section{7}

\section{EFFECT OF RIZATRIPTAN AND OTHER ACUTE THERAPIES IN MIGRAINE: AN OPEN STUDY ON PATIENTS CONSULT- ING ITALIAN NEUROLOGISTS (EMIKRONOS)}

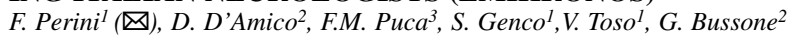

${ }^{1}$ S.Bortolo Hospital, Vicenza, Italy; ${ }^{2}$ C. Bestal National Neurological Institute, Milan, Italy; ${ }^{3}$ University of Bari, Bari, italy

EMIKRONOS is an educational and scientific programme on migraine, aimed to increase awareness and information about this disorder in physicians and in patients. The present study involved a group of about 1000 Italian neurologists. They took part in various meetings about the impact of $\mathrm{M}$ and its treatment; then they were invited to recruit 3 migraine patients (IHS criteria) in an open study. Each patient was given acute therapy and a diary card. Headache intensity at the onset and after 2 and 4 hours from dosing, and functional disability in work and non-work activities during headache attacks were recorded. The main outcome measures were: percentage of attacks with pain-free response within 4 hours recurrence of headache in the 24 hours after dosing; reduction of functional disability during headache (using a 3-point scale: normal functioning; reduced functioning; activities impossible).

Patients included were 1007 (77\% women, 23\% men; mean age 37 years). A total of 2606 attacks were treated; 294 were excluded from analysis. In 1435 attacks rizatriptan was used; in 295 other triptans (103 sumatriptan, 182 zolmitriptan), in 27 attacks ergots, and in 555 analgesics/NSAIDS were used. Percentages of painfree attacks at $0-4$ hours in different therapy groups were: $81 \%$ for rizatriptan; $77 \%$ for other triptans; $67 \%$ for ergots; $60 \%$ for analgesics/NSAIDS. Recurrence rates were: $30.9 \%$ for rizatriptan; $36.9 \%$ for other triptans; $37 \%$ for ergots; $40 \%$ for analgesics/NSAIDS. Statistical analysis (chi-square test) showed similar results as far as both pain-free response and recurrence rate were analyzed (rizatriptan and other triptans vs ergots and analgesics/NSAIDS, $p<0.01$; rizatriptan vs other triptans, $p<0.05)$. Reduction of headache-related functional disability was evident in all therapy groups. Patients receiving rizatriptan showed a higher proportion of attacks in which patients were returned to normal functioning, and a lower proportion of patients who had to stop their usual activities, both in work and non-work domains.

Our results showed that rizatriptan was more effective than other acute migraine therapies (including other triptans) in eliminating pain and in reducing headache-related disability in a large series of Italian migraine patients. EMIKRONOS programme was supported by a grant from Merck Sharp \& Dohme Italia.

\section{8}

QUANTIFICATION AND CONVERSION IN MONETARY UNITS OF THE WORKING RECOVERY OF PRODUCTIVITY IN THE POPULATION WITH MIGRAINE IN THERAPY WITH RIZATRIPTAN

M. Evangelista ${ }^{1}(\varangle)$, D. Camaioni ${ }^{2}$, G. Bussone $e^{3}$

${ }^{1}$ Institute of Anesthesiology, Reanimation and Pain Therapy, Catholic University, Rome, Italy; ${ }^{2}$ Headache Centre, C. Besta National Neurological Institute, Milan, Italy

The Italian version of the Migraine disability assessment questionnaire (MIDAS) was administered to a population of workers with headache, not treated previously.

The aim of the study was to investigate the working recovery of productivity deriving from the administration of $5 \mathrm{mg}$ rizatriptan, for migraine crises. We quantified, in monetary units, the changes in responses to the first two questions of the MIDAS. We carried out descriptive analyses to identify median and percentiles, relative to the time of recruitment (T0), to that of control (T1) and to the variability in the improvements obtained in the two times.

The data deriving from the present study document a recovery of the last working productivity from a median of $\$ 174.42$ to $\$ 1363.86$, with a median improvement of $\$ 1189.44$. The $25^{\text {th }}$ and $75^{\text {th }}$ percentiles were $\$ 523.26$ and $\$ 2270.67$, respectively. Moreover, there was a reduction in indirect costs and a consequent recovery of resources for alternative use. 
ZOMIG NASAL SPRAY, AN ATTRACTIVE ALTERNATIVE TO ORAL DOSING FOR THE ACUTE TREATMENT OF MIGRAINE

G. Montagnani (四)

Medical Department, Astrazeneca Italy, Basiglio (Mi), Italy

Intranasal (IN) administration of acute treatments for migraine is a good alternative to oral dosing. Absorption via the richly vascularised nasal mucosa is faster than via the gastrointestinal mucosa, and can offer quicker pain relief. IN is also unaffected by gastrointestinal symptoms of migraine (nausea, vomiting, gastric stasis), which reduce or delay the absorption of orally administered drugs. IN may therefore be particularly beneficial in those who suffer nausea and vomiting. In addition, IN is suitable for patients who dislike tablets. An IN formulation of zolmitriptan $(\mathrm{Z})$ has been developed and tested in clinical trials.

Methods The clinical programme comprised 5 pharmacokinetic trials, an efficacy and dose-comparison trial (IN doses from 0.5 to $5 \mathrm{mg}$ vs. placebo and oral $\mathrm{Z}$ across 3 migraine attacks), and a 1-year long-term efficacy and safety trial.

Results $\mathrm{Z}$ undergoes IN absorption, appearing in the plasma faster than after oral dosing ( $50 \%$ of $C_{\max }$ within $15 \mathrm{~min}$ ). Plasma concentrations are sustained for 4 to $6 \mathrm{~h}$ after dosing. IN-absorbed $\mathrm{Z}$ avoids first-pass liver metabolism, so the appearance of the active metabolite $183 \mathrm{C} 91$ is delayed. In all other respects, IN $\mathrm{Z}$ is pharmacokinetically similar to the oral tablet. All doses tested $(0.5,1.0,2.5$ and $5.0 \mathrm{mg}$ ) were significantly more effective than placebo in all endpoints, including the primary endpoint of headache response at $2 \mathrm{~h}$. The 5.0-mg dose efficacy was superior and the onset of effect faster compared with the oral 2.5-mg dose, with significantly greater headache response at all time-points from $15 \mathrm{~min}$ to $2 \mathrm{~h}$. This dose was also more effective in attacks with pre-treatment nausea, and against photophobia and phonophobia. Therefore, the optimal dose of IN Z is $5 \mathrm{mg}$. IN Z was well tolerated at all doses. No unexpected adverse events were seen. ECGs or laboratory findings, compared with oral dosing, and local tolerability in the nasopharynx were good. There was no qualitative or quantitative change in events reported during long-term dosing.

Conclusions These results clearly support the use of IN $\mathrm{Z}$ at a dose of $5.0 \mathrm{mg}$ for the acute treatment of migraine with or without aura.

\section{0}

PATIENT GAIN FROM THE ACUTE TREATMENT OF MIGRAINE ATTACKS. ELETRIPTAN VS. SUMATRIPTAN T. Catarci, F. Baldinetti (凷

Pfizer Italy, Rome

Objective To estabilish the actual benefit to patients and clinical relevance of statistically significant differences of efficacy of eletriptan over sumatriptan.

Methods Efficacy data on headache and accompanying symptoms, based on pooled data of 3 clinical trials of eletriptan vs. sumatriptan and placebo involving 2282 subjects $[1,2]$, were expressed as percentage difference of eletriptan over sumatriptan and reported in the order of eletriptan 40 and $80 \mathrm{mg}$. Patient gain was expressed as percentage of patients who regain their functionality after 2 hours of drug intake and patients acceptability of the drug.

Results Pain-free at 2 hours: differences over sumatriptan $50 \mathrm{mg}$ : 8\%*, 15\%* $\left({ }^{*} p<0.03\right)$; over sumatriptan $100 \mathrm{mg}: 6 \% * ; 13 \% *(* p<0.03)$. Sustained pain free: over suma $50 \mathrm{mg}: 7 \% * ; 12 \% * *(* p<0.05, * * p \leq 0.0001)$; over suma $100 \mathrm{mg}: 7 \% *$; $12 \% * *(* p=0.002, * * p \leq 0.0005)$. Accompanying symptoms at 2 hours: photophobia and/or phonophobia-free: over suma 50: $8 \% *, 14 \% * *$; over suma 100: 7\%**, $13 \% * *(* p \leq 0.05, * * p<0.01)$; nausea-free $14 \% *$ difference of ele $80 \mathrm{mg}$ over suma $100 \mathrm{mg}\left({ }^{*} p<0.01\right)$. Functionality and acceptability: more patients on eletriptan 40 and $80 \mathrm{mg}$ could go back to their activities 2 hours after dosing, than those on sumatriptan 50 and $100 \mathrm{mg}: 63 \% *$ and $65 \% *$ vs. $51 \%$ and $48 \%$ respectively $(* p<0.05)$. Finally more patients on eletriptan 40 and $80 \mathrm{mg}$ than those on sumatriptan 50 and $100 \mathrm{mg}$ would take their drug again: $76 \% *, 80 \% * *$ vs. $69 \%, 65 \%$ respectively ( ${ }^{*} p<0.02$ vs. suma $50, p<0.005$ vs suma $100 ; * * p<0.001$ vs. suma 50 and $100 \mathrm{mg}$ ). Conclusions The statistically significant superior efficacy of eletriptan vs sumatriptan, in terms of treatment of headache and accompanying symptoms, has been shown to be clinically relevant because it translates in improved functional response for the patients and better acceptability.

1. Hettiarachchi J (2001) Eur J Neurol 7[Suppl 3]:118

2. Hettiarachchi J (2001) Neurology 8[Suppl 3]:A312

\section{1}

MIDAS QUESTIONNAIRE IN CHILDREN AND YOUNG ADOLESCENTS WITH HEADACHE: A PILOT STUDY

L. Grazzi, D. D'Amico, S. Usai, M. Leone, C. Nespolo, G. Bussone (ख)

C. Besta National Neurological Institute, Milan, Italy
Primary headaches are present in childhood and adolescence. Recently, stardardized methodologies to investigate the global impact of illness have been employed in primary headaches. Marked functional disability and decrease in quality of life have been reported in adult headache sufferers. Specific instruments have been developed to assess headache-related disability. The migraine disability assessment score (MIDAS) is the most studied questionnaire. It is a simple-to-use and a scientifically sound instrument which captures impairment in all activity domains. We performed this study to assess disability in young headache sufferers, using MIDAS questionnaire. The Italian validated translation of MIDAS was used. Testretest reliability of MIDAS scores was evaluated, comparing patient ratings at two different testings, one month apart (Spearman's test). Fifty-nine patients aged 9-16 years completed the study. Diagnosis was made according to IHS criteria. During the first visit, patients were given the MIDAS questionnaire. They were invited to come back to the Headache Centre after one month to complete a second MIDAS form. No change in therapy was performed during the study period. Among the studied patients, 34 had tension-type headache, 18 migraine without aura, and 1 patient migraine with aura; in 6 patients both tension-type headache and migraine were present. Mean MIDAS scores at baseline were: overall, 13.9; item 1, 2.9; item 2, 4.7; item 3, 2.2; item 4, 1.6; item 5, 2.3. Spearman coefficients were: 0.7 for overall score, and $0.6,0.5,0.4,0.2$. 0.6 for individual items $1-5$. Our preliminary results showed that young headache sufferers were disabled in all activities. Reduction in school performance is the most reported problem. A satisfactory test-retest reliability was found for overall MIDAS score, as well as for items which investigate total-partial disability in school performance, and in family/social/leisure activities. Lower reliability coefficients were found in items about household work. These data suggest that MIDAS questionnaire can be used in the assessment of disability in young headache patients, although some changes might be required to have a more suitable instrument for young headache sufferers, namely as far as non-school activities are concerned.

\section{2}

AN EPIDEMIOLOGICAL STUDY OF HEADACHE IN CHILDREN AGED 7-12 YEARS.

L. Sergi (凶), C. Bravaccio, C. Falco, C. Fico, T. Petrarca, G. Testa, R. Militerni Department of Infantile Neuropsychiatry, Second University of Naples, Naples, Italy

The aims of this study were to evaluate the incidence of headache in a population of children referred to a Child Neuropsychiatric Clinic in Caserta and to assess the prevalence of psychiatric co-morbidity. The only inclusion criterion for this study was the presence of headache. The diagnosis was made according to the International Headache Society (IHS) criteria.

Twenty-three consecutive children of both sexes were enrolled between March 2000 and March 2001 from the outpatients presenting at the Child Neuropsychiatric Clinic. There were 14 boys and 9 girls of average age 9.5 years (SD, 2.8). All children received a neuropsychiatric examination and electroencephalogram (EEG). Specific examinations, such as computerized tomography (CT) and magnetic resonance imaging (MRI) were performed on the basis of the clinical findings. We divided the sample into two subgroups according to the reasons of the examination:

- Group I included 9 children referred to the service for "headache";

Group II included 14 children referred to the service for reasons different from headache, but in whom the symptom came out during the examination. On the basis of the IHS, we found different forms of headache in the two groups: in Group I the migraine was the most represented form. The presence of headache is often difficult to assess in young children. The true nature of the symptoms may remain undetermined for years.

\section{3}

EFFECTIVENESS OF AN EDUCATIONAL APPROACH TO MIGRAINE PATIENT

E. Attolini (凶), A. Bassi, M.A. Cassiano, V. Causarano, M.G. Albano, P. Tesauro, V. Centonze

Headache Unit, U.O. Medicina Generale, P.O. Conversano, Bari

Patient education is becoming an increasingly important area of the professional activity of doctors. Our study examines the effectiveness of an educational approach to migraine patients. A course in migraine education was set up for 30 patients suffering from this disease; meetings were structured taking into consideration specific educational aims, with parameters evaluated before the course, at the end of the course, and at 3-month follow-up. We obtained the following results: the increase in the migraineurs knowledge of their disease, the decrease of total monthly numbers of headache days, the decrease of monthly number of medical consultation and biological and instrumental examinations, and a strong reduction of monthly use of symptomatic drugs. Furthermore, our study suggests that there is a need to build educational processes into therapeutics protocols, as they enable patients to manage their chronic diseases more correctly. 